\title{
DISCHARGE FROM THE EAR: OTITIS EXTERNA AND ACUTE OTITIS MEDIA
}

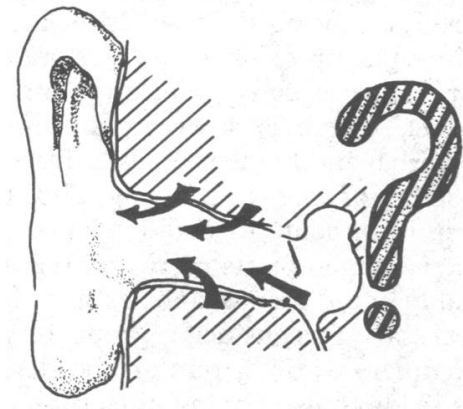

\section{Acute otitis media}

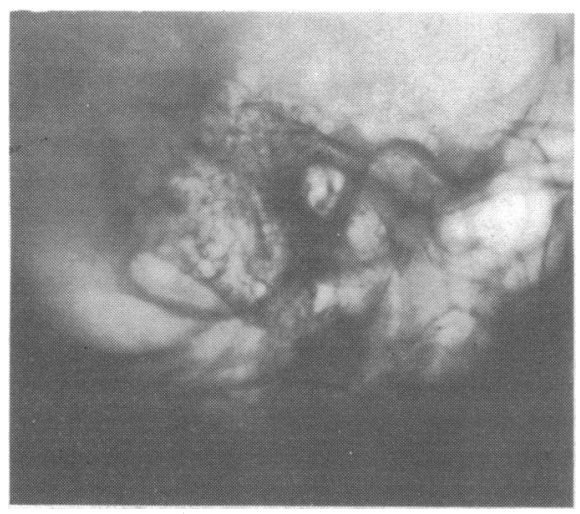

"Subacute" otitis media

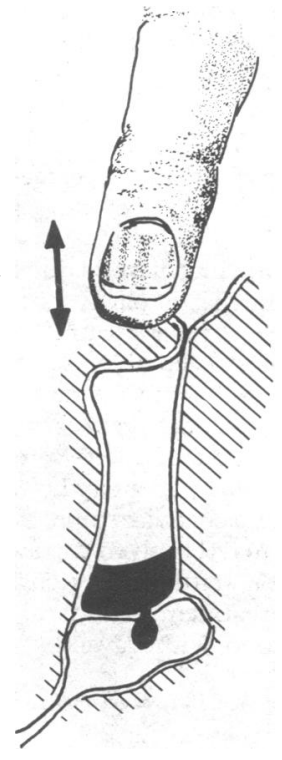

Discharge from the ear occurs in both otitis externa and otitis media. In acute furunculosis and acute otitis media the dominating symptom is pain, which precedes any discharge. Diffuse otitis externa, on the other hand, may present with a thin serous discharge, especially if there is an allergic eczematous reaction. In acute furunculosis the discharge is the thick extrusion of the core of the boil, which consists of necrotic material; there is usually no difficulty in diagnosis.

Discharge in acute otitis media, which is profuse and mucopurulent or purulent, occurs only if the drum perforates. It usually stops within a few days and the perforation heals. Profuse discharge persisting for a week or more may indicate the development of coalescent mastoiditis or a reaction of the mucosa of the middle ear to Eustachian tube inadequacy.

If mastoiditis develops continuing discharge is usually accompanied by continuing ill health and tenderness on deep pressure over the mastoid antrum. The systemic signs may be masked by antibiotics. Mastoid radiographs may help: if they show normal air cells with normal bony trabeculae coalescent mastoiditis can be excluded. When uncertain the only safe course is to explore the mastoid.

Continuing discharge due to mastoiditis is relatively uncommon. More usually the patient, often a child, is well but still discharges mucopus from the ear three or more weeks after a typical attack of acute otitis media. The pattern may arise when grommets are in place in the ear drum. The reasons include continuing irritation of the mucosa of the middle ear by resistant organisms; continuing infection of the nasopharynx, with secondary infection of the middle ear cleft; and changes in the mucosa of the middle ear secondary to Eustachian tube dysfunction of the kind found in secretory otitis media, with more active secretion than normal.

The first step in treatment is to take a swab for culture and to give systemic antibiotics based on the result. After regular gentle toilet to remove infected debris from the meatus, topical antibiotic and steroid drops should be instilled and massaged into the middle ear by pressure on the tragus. Particularly in adults, radiographs of the sinuses may show evidence of infection that needs treating, or there may be infected mucopus in the nose and nasopharynx. Children should be encouraged to blow their noses to prevent mucus from stagnating and becoming infected. Decongestant nasal sprays should be used after blowing the nose for a short period. Systemic antihistamines should be part of the regimen, since allergic swelling of the mucosa around the orifice of the Eustachian tube may be a factor. Provided mastoiditis can be excluded such measures can be used without fear of serious risk for a few weeks. If discharge continues referral is advisable. 

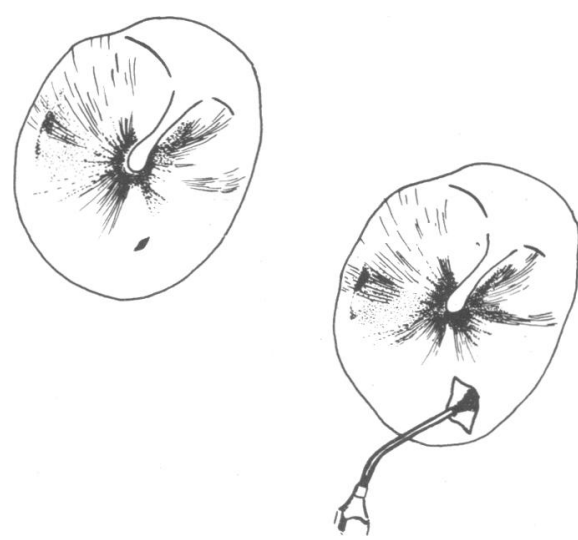

Further measures include removing enlarged adenoids and examining the ear under anaesthetic. The perforation in the drum may be enlarged to improve drainage, while mucoid material can be sucked from the middle ear under anaesthetic. Very rarely continuing discharge may suggest that the mucosa throughout the mastoid air cell system has become secretory, which demands a cortical mastoidectomy to remove the mucosa.

\section{Chronic otitis externa}

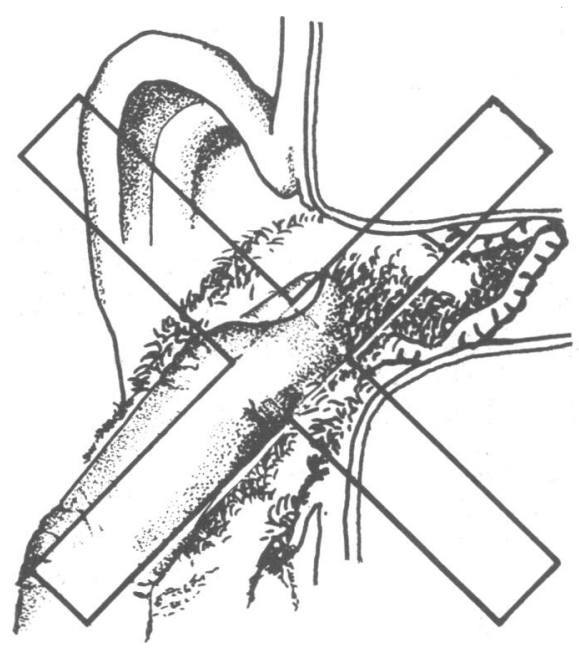

In chronic otitis externa discharge is accompanied by itching, irritation, and impaired hearing. The discharge is often thick, smelly, and composed of infected wax and desquamating skin. The organisms are generally Gram-negative. Nevertheless, the ear drum is normal and there is no conductive hearing loss. The condition is almost always bilateral.

Unilateral otitis externa suggests middle ear disease: there may be a tiny perforation, not easily visible on examination, and the otitis externa may then be secondary to irritation caused by the material from the middle ear.

Chronic otitis externa is partly due to intrinsic factors such as skin diseases-eczema, seborrhoeic dermatitis, or psoriasis - and partly to extrinsic factors in the form of trauma from cleaning the ears, softening the skin by moisture when bathing, or using a dirty towel. There is no better way to produce otitis externa than to bathe in polluted water and then clean the ear with the corner of an abrasive towel that has collected organisms from other parts of the body.

\section{Treatment}

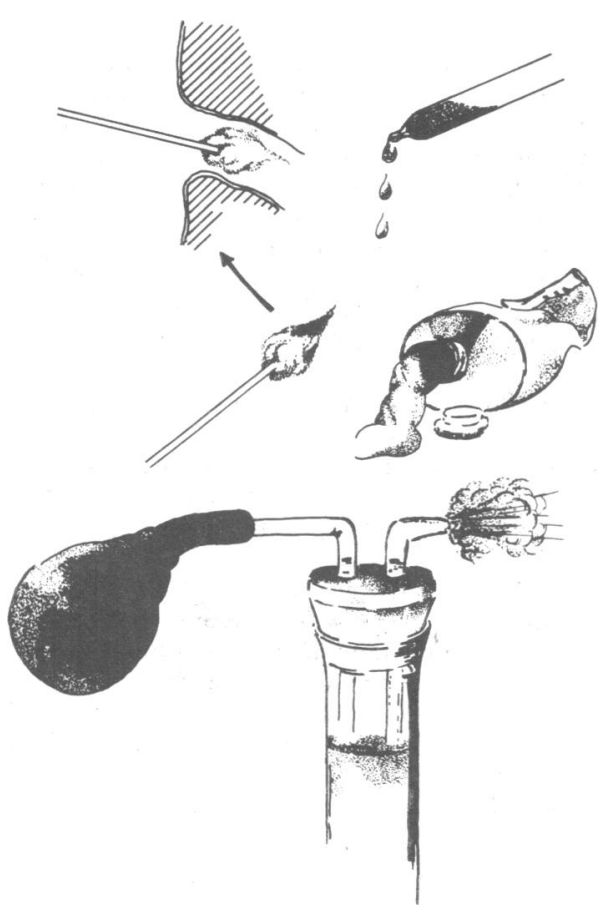

Chronic otitis externa is treated by aural toilet and by instilling topical medication. Careful cleaning to remove infected debris must be done under good illumination with cotton wool on a wool carrier, or by suction under a microscope, paying particular attention to the anterior recess. Provided the drum is intact, gentle syringing can be useful. Toilet should be repeated often-ideally every day; in practice it is usually done once a week. A swab should be taken for culture of fungi as well as bacteria. Useful topical applications include combinations of antibiotics such as gentamicin and neomycin with a steroid. If fungi are present antifungal agents should be used. The medication may be instilled as drops twice a day, painted on the meatal walls with cotton wool, or insufflated as a powder after toilet.

Systemic antibiotics are unnecessary. Eczematous reactions of the pinna may occur and should be treated with ointment. Allergic reactions to topical applications show themselves by worsening of the condition with redness and profuse watery discharge from the pinna. Topical preparations should not be used for long.

When intrinsic factors predominate cure may be impossible though the condition can usually be alleviated. Rarely subepithelial fibrosis causes gross narrowing of the meatus and the lack of ventilation exacerbates the condition. Surgery to widen the meatus is then needed. All patients must be warned to protect their ears from water.

Mr Harold Ludman, MA, FRCS, is consultant otolaryngologist, King's College Hospital, and neuro-otological surgeon, National Hospital, Queen Square, London. 